\title{
Devenir (écrivain), identité virtuelle. Une lecture de Le Prince Ehtejab de Houchang Golchiri
}

\author{
Parisa Lahouti \\ Université d'Ispahan \\ Papa Samba Diop \\ Université Paris-Est Créteil
}

«Tout s'est fait en nous parce que nous sommes nous, toujours nous, et pas une minute les mêmes.»

(Diderot in Barthes 1980: 147)

\section{Introduction}

La problématique considérée dans la présente étude porte sur la transformation de l'œuvre romanesque en autobiographie. Si dans un dialogue chacun des interlocuteurs a ses propres positions, celles-ci ne sont pas figées : elles changent en fonction de l'opportunité et des circonstances. Chaque interlocuteur est alternativement en position d'auteur et de lecteur.

Pour le lecteur, l'écriture autobiographique, selon les conventions de la codification générique, doit constituer un discours de la réalité objective, de la référentiation historique vérifiable. Mais la " Nouvelle Autobiographie » ${ }^{1}$ exige l'abolition préalable de l'ordre ancien et des codes traditionnels du genre fondé sur l'illusion du réel.

La non-assimilation du sujet de l'énonciation permet (au Nouvel Autobiographe) de diriger le discours d'un statut autodiégétique à une plus large homodiégèse. [...] Par un jeu sur la personne centrale et charnière de la narration, non seulement il rétablit une polyphonie, mais il réinstaure par surcroît un rapport de fictionnalité entre l'auteur et le personnage. (Allemand 1997: 172)

En effet, la narration impersonnelle et la confusion pronominale créent une distance entre le sujet écrivant et son propre personnage passé. Esthétiquement, l'une des caractéristiques les plus remarquables d'une telle

\footnotetext{
${ }^{1}$ L'étiquette générique est utilisée pour la première fois par Robbe-Grillet (1994: 17). Ce nouveau genre littéraire a été aussi mentionné comme une «anthropologie», une «entreprise auto-hétéro-biographique ( Ibid.: 190) ou un «pacte problématique de l'autobiographie » émergé des « romans d'apprentissage » ou en général, le « roman des origines et les origines du roman », transfusé en histoire de devenir écrivain. C'est ce que Genette résume à propos de À la recherche du temps perdu par la formule « Marcel devient écrivain » (Genette 1972: 237).
} 
écriture est que son système narratif est composé à la fois d'une sentence narrative inachevée et d'une multitude de segments supplémentaires. Car, comme le dit Barthes, c'est à partir de cet " élément de trop» (issu d'une vision fantasmatique) que le récit (re)commence. Barthes écrit à ce propos que cet élément $[\ldots]$ s'ajoute $[\ldots]$ à [1']ensemble (rhétoriquement) fini [des figures] divisées [et que] l'objet [de] parole [...] est la position du narrateur [...] caché dans l'embrasure, mitoyen au dehors et dedans [et que ce] trouble [...] vient, non d'une carence, mais d'un excès: il y a un élément de trop, et ce supplément indu est le corps (du narrateur). Comme supplément, le corps est le lieu de la transgression mise en œuvre par le récit: c'est au niveau du corps $[. .$.$] que les deux inconciliabilia de l'Antithèse (le dehors et le$ dedans, $[\ldots]$ la mort et la vie) sont appelés à se rejoindre $[\ldots .$. . C'est par ce trop $[\ldots]$, que quelque chose peut être raconté et que le récit commence. (Barthes 1970: 30-2)

Comme toutes les figures des récits modernes, le narrateur du Prince Ehtejab (1968) ne peut pas être défini comme un personnage ou un type d'humain. Aussi le Prince Ehtejab, le personnage central de l'œuvre de Houchang Golchiri (1937-2000), tente-t-il de recréer son ancien monde à partir de ses souvenirs ambigus et des témoignages fragmentés des autres. Comme il n'a pas d'autre choix pour se connaître, son ultime issue est de recréer son passé, même peu réel, à travers les dits de son entourage souvent imaginaire. Le narrateur-auteur cherche à retrouver sa place perdue dans le royaume des fantômes. De même, le lecteur essaie de reconnaitre le narrateur inconnu mais omniscient, à partir du jeu de sa présence/absence. En effet, toute l'histoire est présentée comme une transmission des événements dont le narrateur a été témoin. Par ailleurs, la présence constante de Fakhronessa, l'épouse morte du Prince, dans l'esprit de celui-ci, ne justifie pas l'absence du Prince en tant qu'auteur ou même narrateur. Car l'histoire de Fakhronessa est racontée en rapport avec le récit du Prince-narrateur sous l'apparence de ses témoignages directs ou indirects. Cependant les efforts du Prince pour attirer l'attention de Fakhronessa et ainsi accéder à l'esprit de celle-ci, sont voués à l'échec. Ce n'est qu'à la dernière scène du roman que l'effet d'« ensemble», ce que Lejeune nomme l'«exorcisme d'échec $»^{2}$, se montre au moment où le Prince apprend la nouvelle de sa propre mort de la bouche de son vieux cocher familial à travers le bruit des roues du fauteuil roulant de ce dernier sur le tapis poussiéreux de sa chambre. L'auteur scelle par-là sa propre mort. Il s'agit de l'affirmation du narrateur à la dernière page du récit qui évoque le fait que l'œuvre littéraire n'existe que dans le rêve que l'on nourrit à son propos. L'œuvre qui transmue le présent en passé, constitue bien ce qui est censé répondre à la menace de mort de la littérature à laquelle le narrateur s'efforce de s'opposer. Dès la

${ }^{2}$ Ce que Lejeune dénomme l'« échec spectaculaire de [l']autobiographie [...]. Quand [on] parle d'échec, il ne s'agit pas de porter un jugement de valeur sur [le texte autobiographique], mais de [...] constater [que l'autobiographe a] choisi de laisser [son] autobiographie incomplète, fragmentée, trouée et ouverte» (Lejeune 1996: 42-3). 
première scène où le Prince tuberculeux se trouve tout seul derrière les portes fermées, assis sur son vieux fauteuil, front et les mains appuyés sur sa canne, jusqu'à la scène finale où il se trouve toujours dans le même état (voir Golchiri 1990: 11-2 \& 115), tout le récit est consacré à la présentation du Prince et de ses pensées. Le récit s'effectue soit sous l'apparence d'une voix narrative impersonnelle ou bien par une voix plus personnelle se substituant à cette dernière.

L'expérience d'une illégitimité à accéder au statut d'écrivain constitue un point fort de la mise en récit de l'itinéraire de Golchiri. Cette nouvelle identité s'actualise dans des figures inverses mais symétriques: celles de l'imposture et de l'insuffisance. La démarche que nous avons suivie consiste à différencier ces deux types de figures. Si le sujet peut passer pour un écrivain, selon l'antimodernisme barthésien, c'est la preuve de l'effondrement de la valeur de la littérature ${ }^{3}$. Mais quand l'écrivain n'est qu'un imposteur, copiant les postures de ses modèles sans jamais accéder à une identité véritable d'écrivain, c'est que la littérature n'est plus qu'un jeu d'ombres; un univers qui ne peut se reconstruire que par la substitution d'un passé ignoré par l'œuvre, soit la substitution anticipée du passé au présent, de l'absence à la présence. D'où la construction — dans la fiction et à titre d'exorcisme de l'échec et de la mort d'un sujet sacrificiel qui sauve la littérature en abandonnant l'œuvre au profit de son imposture et de son oubli de soi. La transformation de l'œuvre romanesque en autobiographie, qui est la problématique de notre travail, exige d'évoquer des questions concernant l'identité narrative et la notion de temps comme étapes de devenir-écrivain: dans quelle mesure, un discours monophonique dans lequel l'auteur n'entend pas la voix d'autrui, peut-il devenir la matière première de l'œuvre littéraire? Comment la notion de "temps" devient-elle l'opérateur du récit de vie?

\section{Figures de l'insuffisance: du temps et de l'absence du temps}

Golchiri présente l'autobiographie comme le fil conducteur de ses œuvres. Il s'oppose aux critiques qui considèrent Le Prince Ehtejab comme un roman historique :

On m’a reproché de retourner à l'époque Qajare et négliger le présent; mais moi, je n'ai jamais parlé de cette époque: Le Prince Ehtejab est l'écriture du présent, une écriture dans le temps. Je parle de nos origines ${ }^{4}$ [ainsi que de mon] expérience vécue [et de mon] intériorité. (Golchiri 1999: 819-20)

\footnotetext{
${ }^{3}$ On peut ainsi soutenir sans doute que chez Golchiri, le renoncement à la création en faveur de l'imposture est ce qui soutient la valeur menacée de la littérature.

4 Voir Golchiri (1999: 794-5), où il explique que Fakhronessa est une meilleure
} 
En effet, lisant Lejeune ${ }^{5}$, on peut se demander si le Nouvel Autobiographe, en écrivant son "récit de vie" n'a pas plutôt la volonté de refuser l'idée lejeunienne et de se dissimuler derrière la fiction, l'écriture ironique ou les mises en abyme. Le postulat de sincérité ou le souci d'authenticité est ainsi de plus en plus en péril. Certes, la sincérité consiste moins à dire la vérité qu'à dire ce que l'on pense ou ce que l'on ressent. Rappelons ici la célèbre phrase de Barthes : "Voici une suite de propositions [...] contradictoires [...]: je ne serais rien si je n'écrivais pas. Cependant je suis ailleurs que là où j'écris. Je veux mieux que ce que j'écris » (Barthes 1975: 172). Golchiri confirme ce qu'avance Barthes ainsi : "si on accepte le contrat de "la vérité sensible", donc la partie créatrice de l'écrivain doit se trouver plus dans les apparences de son écriture (ce qui se voit et se sent directement) »(Golchiri 1999: 702). Dans Si le grain ne meurt, le jeune Gide explique ainsi son désir de devenir écrivain: Le plus gênant, c'est de devoir présenter comme successif des états de simultanéité confuse. Je suis un être de dialogue; tout en moi se combat et se contredit. Les Mémoires ne sont jamais qu'à demi sincères [.] Peut-être même approche-t-on de plus près la vérité dans le roman » (Gide 1991: 280).

Ainsi les éléments de la mémoire (souvenirs et fantasmes) présentent des qualités de fragmentation et de mobilité qu'il faut s'efforcer de préserver pour conserver toute la vitalité de l'existence et ne pas composer un autoportrait figé pour toujours en une image artificielle. Or, la perspective à la fois historique et contemporaine dans l'œuvre autobiographique golchirienne ainsi que son langage archaïque et harmonieux peuvent être considérés comme des signes initiaux du devenir écrivain. Todorov écrit à ce sujet :

Il ne faut pas croire que l'histoire correspond à un ordre chronologique idéal. Il suffit qu'il ait plus d'un personnage pour que cet ordre idéal devienne extrêmement éloigné de l'histoire "naturelle". La raison en est que, pour sauvegarder cet ordre, nous devrions sauter à chaque phrase d'un personnage à un autre pour dire ce que ce second personnage faisait "pendant ce temps-là". [...] elle contient le plus souvent plusieurs "fils" et [...] ces fils se rejoignent. (Todorov 1981a: 133)

Les déplacements métaphoriques dans Le Prince Ehtejab finissent par la représentation des deux espaces : la maison ancestrale et la nouvelle maison. L'espace enfantin de la maison ancestrale où le Prince fait une grande partie de sa carrière, est caractérisé par son absence de souvenirs. Le Prince ne se souvient pas de son enfance. Tout ce qu'il se rappelle de cette époque-là n'est que ce qu'en dit son entourage. Le second espace, la nouvelle maison, est celui

représentation des miniatures persanes.

${ }^{5}$ «S'ils [Gide et Mauriac] n'avaient pas écrit et publié aussi des textes autobiographiques, même "insuffisants", personne n'aurait jamais vu de quel ordre était la vérité qu'il fallait chercher dans leurs romans » (Lejeune 1996: 42). 
où le Prince a enfermé Fakhronessa et sa bonne, Fakhri. Or, comme il est bien indiqué dans le texte, l'auteur ne considère pas de diversité spatio-temporelle ni narratoriale : "On ne donnait pas d'eau aux prisonniers, seulement quelques gorgées chaque jour, afin qu'on ne les déplace pas continuellement [...]. Seulement un morceau de pain. On l'avait jeté dans une des pièces communes pour qu'il fasse des aveux au sujet de ses autres biens » (Golchiri 1990: 105-6).

C'est bien là l'exposition de l'enfermement du "je" narrateurautobiographique, présenté à partir de son absence et de sa tentative de se libérer, à travers ses mouvements labyrinthiques entre l'absence et la présence ainsi qu'à travers la formation de l'écriture stéréotypée.

Peut-être tout discours monophonique et non objectal est-il naif et inapproprié à la création authentique. La voix authentique créatrice ne peut jamais être qu'une voix seconde dans le discours. Seule la seconde voix [...] peut rester jusqu'au bout non objectale [...]. L'écrivain est celui qui sait travailler le langage en étant lui-même hors du langage, celui qui possède le don de la parole indirecte. (Todorov 1981b: 106)

Le Prince se prête ainsi au jeu social. Après sa sortie de la maison ancestrale à laquelle il était attaché, il passe tout son temps hors de la maison, à jouer aux cartes ou à boire du vin pour échapper au regard attentif de Fakhronessa. Cette dernière le sait et elle veut que les autres aussi soient au courant de cet échec. Comme elle se souvient de son père qui préférait sa solitude, après sa retraite, après la rupture avec sa femme, et même après sa sortie de la prison princière : «Il n'avait que la peau et les os. Les marques du fer et de la chaîne étaient restées sur ses mains et ses pieds. [...] Le grand-père ne lui rendit pas ses propriétés, mais seulement la maison » (Golchiri 1990: 87-8). Certes, il y a un jeu social, mais y a-t-il un "je" sociable? En fait, on a une meilleure figure de l'emprisonnement du Moi. Barthes l'explicite ainsi : " ce supplément de message ne doit altérer en rien [...] l'essence précieuse de [s]on individu: ce qui [est] en dehors de toute effigie » (Barthes 1980: 26-7). L'auteur résume ainsi la trame principale de son autobiographie en absence de son moi : «le Prince se tenait dans l'obscurité [...] je m’approchai de lui. [II] ne se retourna pas» (Golchiri 1990: 68).

Par ailleurs, dans cette nouvelle maison, le Prince-narrateur a deux prisonnières qui ont des caractéristiques différentes, et même contradictoires : la maitresse Fakhronessa et sa bonne Fakhri. Comme des âmes errantes, ces personnages n'en finissent pas de faire du surplace. En réalité, leurs déplacements se bornent à l'intérieur d'un espace qui se réduit qu'à une pièce obscure et close. Dans des romans qui ont pour objet de faire l'analyse de ce qui se passe dans la tête du narrateur, il est logique que l'espace soit formé audelà du monde factice de la quotidienneté, celui de la vie dite consciente, de 
l'univers qu'affronte notre inconscient, qu'il soit conforme à cette réalité où tout est double et contradictoire.

$[\ldots]$ cette maison $[\ldots]$ je l'ai choisie à la vue des murs. Je me suis dit qu'il valait mieux faire abattre le saule [...]. J'avais ordonné qu'on apporte les provisions à la maison [...] afin qu'elles n'aient rien à faire dehors. [...] Fakhri disait: "[...] Madame marche sans cesse autour de la cour. [...] Quelquefois, quand elle toussait, elle allait s'asseoir sur sa chaise, sous le saule $6 . "$ (Ibid.: 107-8)

Dans l'esprit du Prince, Fakhronessa est cette accusée qui est toujours présente mais toujours inaccessible. À l'inverse, l'autre prisonnière, Fakhri se plaint simplement d'agir comme l'espionne de Fakhronessa et de l'imiter sans cesse. Comme le narrateur du récit — qui lui-même joue le rôle de l'espion des personnages - elle n'est qu'un rapporteur déficient qui ne sait rien de ce qui se passe hors de son regard : "Madame [...] a dit : [...] "Fakhri, on s'ennuie dans cette maison, avec toutes ces pièces [...]. Si seulement le Prince achetait une autre maison. Cette demeure est devenue vieille. Toi non plus tu ne peux pas t'en occuper toute seule" » (Ibid.: 102-3). La vision stéréotypique présentée dans cet ouvrage permet de voir les personnages à la fois de près et de loin. Fakhronessa est à la fois présente dans la pensée du Prince et attachée à son passé. En revanche, Fakhri est proche du Prince et indépendante par rapport au passé. Ces deux personnages ne sont pas complémentaires l'un de l'autre. Métamorphosée par le Prince, Fakhri remplace sans interruption Fakhronessa et comble apparemment le vide laissé par cette dernière, tout en étant en forte opposition par rapport à elle. Les saisies de près et de loin du phénomène créatif sont ainsi confrontées. Dans la saisie de près ou la saisie au présent, l'acte créateur se donne pour improbable et insaisissable tel que nous a appris aussi à le voir la modernité. Il échappe tout à la fois à ce qui l'accomplit et à ceux qui le voit s'accomplir au présent. La saisie de loin aussi permet de mettre en scène la saisie de l'œuvre comme passé. Le regard a posteriori du Prince à travers les mémoires de ses ancêtres, fait la valeur de l'œuvre et redouble l'expérience de l'échec du Prince dont Fakhronessa est le témoin: "Mais Fakhronessa ... C'était comme si dans l'ensemble de ces lignes du coin des lèvres, il y avait quelque chose qui faisait peur. On sentait combien on était petit et insignifiant, même si on est le petit-fils de son Altesse. Je voudrais mourir » (Golchiri 1990: 93).

\footnotetext{
${ }^{6}$ Le saule, l'arbre qui a une grande valeur en littérature classique persane, accompagne Fakhronessa à plusieurs reprises. La convergence entre ces deux compagnons se trouve dans leur attachement au passé: Fakhronessa représente l'image d'un monde perdu, sa disparition/réapparition successive dans la pensée du Prince met le temps en suspens dans la collusion passé-présent. Toutefois, le saule est amoureux et se plaint sans cesse de la séparation, tandis que Fakhronessa ne parle pas beaucoup. On peut dire donc que le saule est son porte-parole.
} 
Mais c'est plutôt le Temps qui fait peur et fait la différence. «Le temps est engorgé » et cette "immobilisation du temps ne se donne que sous un mode excessif [et] monstrueux » (Barthes 1980: 142). Devant cet "écrasement du temps »(Ibid. 150), devant le visage pâle de Fakhronessa, devant ses yeux morts et donc de plus en plus étranges, devant ses cheveux couverts par la poussière, le Prince n'a plus rien à perdre et il se rend compte qu' "au bout de cette première mort, [s]a propre mort est inscrite » (Ibid. 145). Fakhronessa dit : «Prince, $[\ldots]$ il semble qu'il ne te reste plus rien. [...] j'attends de voir quand tu feras faillite et vendras cette maison. [...] On n'en est pas loin, attends » (Golchiri 1990: 76). Néanmoins le Prince s'interroge toujours sur son attachement à l'image pâlie de Fakhronessa, celle d'un passé en ruine. Il se remémore Fakhronessa lui rappelant : «Vous savez, si nous voulons nous connaître, il nous faut commencer par ces chose-là, par ces ancêtres » (Golchiri 1990: 45). Et le Prince fait une autre fois confiance à son épouse et se remet à la recherche de son passé ignoré. Par l'image éblouissante de la mort, le Prince veut se connaître.

Ainsi, malgré son inaccessibilité, le personnage-lecteur ou l'autre motive le narrateur-auteur à chercher des autres indices pour modifier son existence à l'image du Prince désespéré à la recherche de Fakhronessa. Par l'alternance entre la présence et l'absence du temps, le sujet est transmis aux mots portant les signes du temps. De ce fait, la narration prend une nouvelle direction. Cette fois-ci, le Prince commence par les rappels successifs de morts de sa famille :

Morad Khan [...] fouettait les chevaux dont les fers retentissaient sur l'asphalte de la rue. » (Golchiri 1990: 22-3) «Le prince Ehtejab, la grand-mère et le père étaient assis sur la banquette de la calèche. [...] C'était la calèche du grand-père. Le cercueil noir, à l'avant, s'agitait au-dessus de la foule. [...] Le Prince dit: "Je veux descendre." "Les chevaux vont t'écraser, dit la grand-mère." [...] Seules la mère et les tantes pleuraient. [...] La grand-mère n'était pas là. Il y avait le père et la mère. (Ibid. 39-41)

Par la mise en abyme du texte principal et l'apparition et la disparition des phrases, des sentences et des images, les distances spatio-temporelles sont négligées. Les épisodes du deuil se suivent malgré leur décalage et s'expriment en détail. La scène antérieure est l'explication de l'enterrement du grand-père, bien que la phrase suivante renvoie aux funérailles de la grand-mère qui sont suivies par celles du père du Prince: "Mère je veux descendre, dit-[le Prince]." "Tu n'es plus un enfant, mon fils." Elle pleura. [...] Là, devant, le cercueil du père s'agitait. "Tout le monde doit mourir un jour Prince, dit Morad. [...] Le Prince Ehtejab [...] se tint debout jusqu'à ce que cessa le va-etvient de la foule. On enterra le père au pied du grand-père et de la grandmère » (Ibid. 41-2). 
S'intéressant au rapport entre ce qui est raconté et celui qui raconte, l'auteur représente indirectement le décalage des épisodes narrés. D’abord, le petit Khosro du premier épisode des funérailles cède sa place au Prince du deuxième épisode. D'ailleurs, certaines images comme "la calèche » et «le cercueil » appartiennent aux trois scènes du deuil, bien que la présence ou l'absence de certaines personnes indiquent leurs différences spatio-temporelles. La calèche qui a remplacé le cercueil, sera substituée plus tard par le fauteuil roulant. À travers son voyage hors du temps «dans l'allée centrale du jardin» (Ibid. 22), le narrateur va plus loin afin de rester à l'abri du regard attentif de son narrataire. Ainsi se télescopent les sentences divergentes «les chevaux avec les fers aux pieds» et «la rue asphaltée ». Mais jusqu'où peut-on aller? Evidemment, il ne faut pas aller trop loin. Morad est désormais la victime de ce passage hâtif : dès le début de l'histoire, il portait les morts de la famille Ehtejab sur sa calèche; à présent, il prend la parole et devient le porteur de la nouvelle de la mort de cette famille, en montant péniblement les marches avec son fauteuil roulant. L'image transformée de la "calèche»- porteuse des cadavres au "fauteuil roulant», est une métaphore narrative : l'image-temps qui suscite une instabilité des repères spatio-temporels disséminés dans le corps du texte d'une manière apparemment désordonnée de sorte que la présence énigmatique du narrateur-auteur se trouve mise en cause. En réalité, c'est à partir de la coïncidence de l'évocation du "je" autobiographique et de celle des funérailles, c'est-à-dire à partir de l'élimination ou plutôt de l'ignorance de la différence spatio-temporelle, que le texte se développe. En effet, le Nouvel Autobiographe suit Deleuze dans son processus de devenir; car d'après Deleuze, «le devenir » est le débat entre ce qui est loin et ce qui est près (1968: 17). Pour lui, l'autobiographie est une sorte de «dialectique» (Idem.) qui est l'exposition des «séries des événements qui dépendent les uns des autres [...]. Mais [elle] appartient au langage à la fois d'établir des limites et d'outrepasser les limites établies »(Ibid.17-8). En effet, le récit de vie du Nouvel Autobiographe s'achève par sa mort. Comme l'explique Barthes, devant l'« écrasement du Temps: [le sujet] est mort et [il] va mourir» (1980: 150).

\section{Masques de l'imposture: à la recherche de l'union perdue, une présence virtuelle entre le personnage évanescent et le lecteur éventuel}

À partir de l'exigence explicite de la définition du "je" autobiographique pour distinguer le narrateur-auteur de l'autre, le personnage qui le défie, se définit l'identité narrative. La distinction entre le narrateur-autobiographe et le personnage devient extrêmement difficile, car le Prince intervient dans une partie considérable de la narration du texte golchirien, aussi longtemps que son 
histoire semble le fondement de ce texte. Issu du postulat impossible de sincérité de la Nouvelle-Autobiographie, le narrateur devient l'égal de l'autobiographe. Cependant, ce personnage est présenté par des signes qui nous empêchent de lui attribuer le statut de narrateur autobiographique représentant l'auteur. Le Prince vit dans des conditions qu'il ne mérite pas ; il passe sa vie mystérieuse dans des couloirs étroits et impénétrables. Voilà quelques signes de duplicité qui accompagne ce personnage tout au long de son récit. La manière dont il est représenté permet au lecteur de le considérer comme crédible et l'amène à conclure que ce personnage aurait davantage la fonction d'opérateur textuel. Pourtant, même si nous en arrivons à l'identifier selon ce qui est dit dans le texte, il présente tellement d'aspects contradictoires et incertains que nous ne l'acceptons que comme figure romanesque.

D'ailleurs, on peut peut-être imputer ces indices d'incertitude à l'imagination du narrateur qui n'a pas vraiment connu son héros : « Khosro [à] qui le $[\ldots]$ grand Prince $[\ldots]$ disait : "Mon fils, j'espère que tu ne deviendras pas indigne, comme ton père" [...] a attrapé la tuberculose. Il était devenu maigre comme un clou. On ne pouvait plus le connaitre » (Golchiri 1990: 1145). A travers une représentation fragmentaire, le narrateur transfère le caractère d'invalide du personnage à son lecteur éventuel.

Condamné à vivre pour toujours et hors du temps, le Prince est attaché au vieil espace familial et tyrannique où tout le monde a attrapé la tuberculose et est obligé de rester silencieux. De plus, sa mort mystérieuse, prévue depuis le début et annoncée ironiquement avant sa réalisation, est l'autre signe de duplicité de ce personnage : "Morad dit : "Cher Prince, le Prince Ehtejab vient de mourir." Le Prince demanda: "Ehtejab?" Morad dit : "Vous ne le connaissez pas? [...] Je parle de Khosro» (Golchiri 1990: 114). Ce personnage fuyant se mêle souvent au narrateur dont la présence est perpétuelle, mais l'identité incertaine. En effet, les informations ainsi prodiguées sur le journal intime d'un autobiographe demeurent soumises à un point de vue particulier : le Prince apparait bel et bien comme un double de l'auteur, un miroir de l'autobiographe à l'intérieur de son autobiographie. Son histoire racontée à partir d'une narration partielle est transformée en un espace fantasmatique où les personnages fuyants se déplacent perpétuellement. La construction du personnage à partir du manque de cohérence et de l'abandon de légitimité, exige un nouveau "morcellement», pour reprendre l'expression barthésienne (1970: 110).

C'est là que commence l'avenir, le devenir (artiste). L'évocation d'un corps morcelé s'achève par son rassemblement : la bouche expressive va désormais être calme; les yeux amoureux ne verront plus rien; la tête aura du vertige à 
force d'ivresse, le visage prendra une blancheur éblouissante et finalement les oreilles n'entendront qu'une voix connue depuis toujours, mais arrivée tardivement. Il n'existe plus de distance entre ce corps « morcelé-rassemblé » et son modèle. Et c'est là le plaisir d'atteindre le désir d'être accepté comme il est.

L'auteur aussi, comme son lecteur, reconnaît son personnage par endroits, comme s'il s'agissait d'un fantôme privé d'ancrage spatio-temporel. Son histoire est la répétition de celle de ses ascendants tuberculeux et agonisants. Le Prince enferme Fakhronessa (la femme angélique) dans une maison avec de grands murs. Elle souffre puis meurt d'une tuberculose dans cette petite maison. Dans tout le récit, il y a aussi plusieurs scènes de torture de Fakhri perpétrée par son maître, le Prince. Ce dernier, comme ses ancêtres, jouit de torturer les «jolies filles» pour reprendre l'expression sadienne, alors qu'il le dénie. Fakhri, forcée d'imiter sa maîtresse, perd son identité (voir Golchiri 1999: 716). "Le Prince me frappa le visage et cria: "Chère Fakhronessa, toi, tu n'étais pas comme ça." Je dis : "Je ne suis pas Fakhronessa, moi !" » (Golchiri 1990: 84). Malgré son manque de naturel et son air absent ou son sentiment d'être en trop dans le monde, privé de savoir et de pouvoir, le Prince tente désespérément d'accéder à une existence charnelle qui lui est refusée, et est entraîné dans sa quête de l'autre, son lecteur.

C'est également l'autre ou le lecteur qui n'a que très peu d'informations concernant le héros et tente désespérément d'entrer dans l'univers de ce héros pour accéder ainsi à son identité inaccessible à l'aide des modèles préalables présentés dans l'écrit. En suivant la narration fragmentaire, suspendue entre le réel et l'imaginaire, le lecteur est déjà entré dans la construction de la structure du texte. "Elle s'asseyait sur la terrasse et lisait. Quand je m'approchai d'elle, [...] elle dit : "Prince, ne reste pas ici sans rien faire. [...] Il faut que tu fasses quelque chose" »(Golchiri 1990: 97). C'est encore à travers l'ambiguïté que l'auteur lui-même essaie d'éclaircir le chemin de sa lecture (Baetens et Viart 1999: 127), en avouant son choix précis de lecteurs.

Seuls les jeux de cartes m'occupaient. [...] Lorsque je voyais que la main de l'adversaire tremblait et que sa paupière inférieure battait [...] C'est pour toutes ces raisons que j'étais attiré par ce jeu. Il fallait bien d'une façon ou d'une autre dilapider biens et propriétés. Quand je n'avais rien en main, je bluffais. (Golchiri 1990: 98)

Pour le Prince, l'objectif est désormais la connaissance de sa lectrice désirée, Fakhronessa : "Sous ce front lisse, que se passait-il? Comment peut-on se mettre à la place de ces yeux, derrière ces verres épais de lunettes me regarder [...]? » (Ibid. 99). Cet autre cherché à travers l'inaccessibilité est Fakhronessa ${ }^{7}$;

7 P. Ricœur divise l'identité personnelle en deux : la mêmeté et l'ipséité. Il l'explique ainsi: « À la perte d'identité du personnage correspond [...] ainsi la perte de la 
le contrepoint à cette déficience est son sa pureté et son innocence ${ }^{8}$ : «Fakhronessa ne battait jamais des paupières, elle feuilletait constamment des livres [...]. Seules ses lèvres bougeaient. » (Ibid. 60) «Je ne l'ai jamais vu avoir la tête tournée. Elle disait: "Prince, pense à ta vieillesse, moi, je suis mourante. » (Ibid:: 74). En effet, en considérant le concept barthésien de la textualité, Fakhronessa est l'absence antérieure à la présence, ou plus précisément, l'innocence et la pureté de la langue dont «la simplicité de la structure et la

configuration du récit et en particulier une crise de la clôture du récit. [...] Ce n'est pas non plus par hasard si maintes autobiographies contemporaines $[\ldots]$ rejoignent $[\ldots]$ le genre littéraire le moins configuré, l'essai précisément. [...] ces cas déroutants de la narrativité se laissent réinterpréter comme mise à nu de l'ipséité par perte de support de la mêmeté. » (Ricœur 1990: 13) Si l'on interprète le roman de Golchiri dans le cadre de la dialectique du "même" et du "ipse", l'on peut le concevoir ainsi qu'il manifeste l' " ipséité » dans sa forme dénudée qui a perdu l'appui de la "mêmeté ». Fakhronessa est la figure de la "perte de support de la mêmeté » dans une pleine ipséité du récit du Prince et ainsi du narrateurautobiographe. Au lieu d'avoir une existence remplie et réelle dans le texte, son existence est formée de multiples stéréotypes et de clichés comportant volontiers des lacunes. L'autre est transformé en une fille angélique dont la présence n'est dérivée que des parcours imaginaires et fantasmatiques du Prince.

${ }^{8}$ C'est ce que Derrida dénomme «la différence » (Derrida 1972: 6) et Merleau-Ponty, « la vertu du langage » (Merleau-Ponty 1969: 16). Cette «fameuse "innocence" désigne à la fois une pureté d'avant le désir et un appétit de jouir point encore formulé ; la sacro-sainte "ignorance", une virginité d'âme [et de langage] d'avant la représentation [,] l'ignorance avant le savoir, la naïveté avant son dévoiement, l'innocence avant qu'il ne soit trop tard » (Cusset 2002: 106). Il s'agit de l'ignorance avant le savoir ; le savoir qui sera tenu comme un complément d'écriture, car il est retenu comme puissance.

${ }^{9}$ L'une des modalités barthésiennes caractéristiques est la «textualité » présentée dans les années soixante. Pour ce qui est de la textualité, il s'agit, premièrement, de la distinction entre l'œuvre et le texte. On peut dire que, si l'œuvre peut être définie en termes hétérogènes au langage, le texte reste de part en part homogène au langage: il n'est que langage et ne peut exister qu'à travers un autre langage. En d'autres termes, le texte est l'étape d'avant l'œuvre, c'est-a-dire qu'il est l'étape langagière d'avant la représentation. Entre l'œuvre et le texte, entre l'avant-garde et le classicisme, Barthes ne cesse jamais de changer et de participer à la dispute de critique littéraire, mais ce qui ne change pas, c'est qu'il se retrouve toujours marginal dans son refus perpétuel de la mode et de l'idéologie dominante. Deuxièmement, d'après cette théorie, il y a une opposition entre le "plaisir" du texte et la "jouissance", dont Barthes valorise le second terme en l'associant à la fragmentation du texte (Barthes 1970: 123-4). La "jouissance" que Barthes met en valeur se fonde sur l'hétérogénéité de l'expérience, tandis que le "plaisir" se base sur l'homogénéité: l'œuvre plaît au lecteur, et la textualité le stimule par la transgression de ce qui a déjà existé. Le "plaisir" est une répétition de la beauté, et la "jouissance" vient de tout ce qui abolit ce stéréotype (Voir aussi Barthes 1973: 25 \& 59-60). D'ailleurs, la «théorie du texte » peut également trouver des spécifications historiques dans l'usage de la "lecture": la lecture réécrit le texte. Non seulement, la «théorie du texte » élargit à l'infini les libertés de la lecture, mais encore elle insiste beaucoup sur l'équivalence (productive) de l'écriture et de la lecture. C'est la lecture seule qui «sauve le texte de la répétition » et lui offre une "jouissance" érotique (Barthes 1970: 22). 
brièveté emporte [une] dénotation [...] dont les autres codes se servent pour adoucir leur articulation » (Barthes 1970: 122-3). Le Prince aussi est ainsi plus précis dans sa division intérieure entre un moi acteur et un moi spectateur, qui interdit la possibilité de toute présentation perceptible.

$\mathrm{Si}$, comme les honorables ancêtres, je pouvais $[\ldots]$ surveiller l'accusé par un espion [!] comment pourrait-on pénétrer au plus profond d'un individu? Ou bien reconstituer sa personnalité? [...] l'accusé et l'espion [,] deux personnes libres au milieu de grands murs [avec] un saule, et quelques centaines de livres? Et moi? Moi ... (Golchiri 1990: 99-102)

La tentative du Prince pour retrouver le temps perdu pousse finalement l'artiste-écrivain à tourner le modèle, la toile ou le texte afin de s'assurer de son intérieur et le conduit à avouer son échec : «Le Prince voyait que Fakhronessa [...] était si loin et méconnaissable. [...] lorsqu'il vit qu'il ne pouvait plus... Il comprit qu'il fallait s'y mettre, qu'il fallait à tout prix ... et il dit tout haut : "Comment?" » (Ibid.: 59).

Le narrateur-auteur sait bien qu'après avoir détruit le portrait illusoire du témoin de son échec, c'est son propre portrait qui s'efface. Le personnage romanesque qui fuit et en même temps cherche sa route, est toujours perçu comme «le moyen d'expression d'une recherche à la fois esthétique et ontologique » (Allemand 1996: 50).

Comme il n’y a pas de simultanéité du langage et de la représentation, le narrateur-auteur est en quête de son identité dont il déconstruit l'image au fur et à mesure qu'il la découvre. Pourtant, cette quête se fonde sur l'énergie de l'écriture, où l'écrivain est hanté par son imagination, ses rêves et son réel, où il insiste sur sa présence, reprenant son chemin vers l'infini; son dernier mot dont la représentation est le texte, referme à la fois son "je" dédaléen sur lui-même et le chemin éventuel des personnages, ainsi qu'il ferme le livre. Cependant, tout doit chanter ensemble pour être présent, car la représentation ne sera possible que dans la réconciliation des écarts: "La toux fit trembler les épaules du Prince. [...] Et il vit que Fakhronessa était allongée de toute sa longueur et le sang était en train de tâcher le drap blanc et de s'y répandre. Il toussa et le sang humidifia sa bouche et le coin de ses lèvres. [...] L'aurore avait éclairci toute la pièce » (Golchiri 1990: 115). Nous sommes ainsi à nouveau rapportés à l'ensemble de l'œuvre, à partir de chaque point d'intensité marqué, de chaque attention portée sur un trait, dans une fuite du regard, vers un bruit ou une couleur. Cette partie n'est qu'un recommencement; elle est commencée et ne finit jamais : «Le Prince [...] constata que Fakhronessa [...] hors de portée de main, existait et n'existait pas. [...] Et à nouveau il entendait le grincement des roues et le pas de [Hosna]. Le fauteuil roulant 
montait l'escalier [...]» (Ibid. 113). Il nous semble que cette fois rien ne roule comme avant, que le monde est désormais différent : l'intérieur et l'extérieur sont unis par les déplacements de lumière et les mouvements des roues du fauteuil roulant de l'homme et le bruit des pas de la femme sur le tapis. Et il n'y a qu'une seule voix qui s'entend et qu'une seule voie qui se voit:

Quand la porte fut ouverte, le Prince n'entendit que le bruit des roues et les pas de la femme. [...] Il cria: "Morad, quelqu'un est encore mort, hein?" Et il toussa. Avec la lumière de l'allumette, le Prince ne vit que ces deux mêmes yeux parmi les rides et la faible lueur de la cigarette. Il savait que maintenant le fauteuil roulant était tout près de la cheminée et que [Hosna] était en train de fouiller dans les cendres. (Ibid. 114)

Mais ce n'est que la voix d'un aveu d'impuissance qui s'entend. Et ce n'est qu'à partir de ce monde infiniment mouvant et parfois immobilisé dans les instants de quasi-abstraction, que l'auteur construit et reconstruit son univers imaginaire unifiant les deux instances de l'écriture et de la lecture. En effet, le barrage entre les deux côtés reste immuable et la communication est interrompue.

Après « la mort de Dieu », le Prince est livré à lui-même et doit écrire son récit de vie dans sa "chambre génératrice » dans une solitude absolue. Car la « chambre créatrice » est le lieu où s'affirme la liberté; et l'artiste n'étant pas en mesure de ne pas évoquer ce lieu libérateur, se libère à son corps défendant. Or, un côté reste constamment inachevé :

Morad dit: "Cher Prince, le Prince Ehtejab vient de mourir." [...] Le Prince entendit $[\ldots]$ le bruit des roues sur le tapis [et puis] sur [...] les marches de l'escalier. [...] Et le Prince [...] descendait de plus en plus bas toutes ces marches qui aboutissaient à ces couloirs humides, cette crypte froide, ce drap, ce sang, et à ces yeux fixés qui étaient et n'étaient pas. (Ibid. 114-5)

La lecture reste curieusement tiraillée : elle est emportée parfois par la tonalité de l'ennui et la vaste structure d'une attente, d'une vie qui rêve, puis se précipite et s'oriente vers une grande aventure, une étrange dissymétrie, des découpages successifs des épisodes. L'histoire contient donc une violence par elle-même : la narration semble quelquefois emportée comme une nécessité, et conduit, en même temps, à la discontinuité de l'histoire. En effet, une telle écriture ne donne pas de liberté d'évasion : dans une telle vision, parmi les vibrations des bruits et des couleurs, on ne lit ce livre que comme un tableau vaguement décrit offrant une vision de la subdivision et de la répétition en chaque point. Le lecteur abusé est ainsi contraint de suivre le chant des ombres.

\section{Conclusion}


Le Prince Ebtejab, comme tous les Nouveaux Romans, s'inscrit dans le contexte d'une évolution de la représentation: l'écriture comme l'expression d'une recherche à la fois artistique et ontologique. Les caractéristiques de ce genre littéraire s'articulent autour d'un axe majeur : il ne peut s'agir de croire à une quelconque authenticité du récit de vie, car le processus de l'écriture est par essence fabulateur. Et c'est là où la mobilité du je empêche d'en identifier le référent, et se saisir soi-même devient impossible. Un tel écrit est donc une interrogation sur la pratique d'écriture de même que l'affichage d'une contradiction majeure dans le travail de l'écrivain : le déni de lecteur, d'une part, et le désir immédiat d'un lecteur indissociable de l'horizon du livre à écrire, d'autre part. Et ces deux objectifs ne seront possibles qu'à travers deux moyens : d'abord, parmi les précurseurs et les écrits antérieurs — «les mémoires ancestraux» — être à la recherche de la place et du rôle de ce lecteur imaginaire et fantasmé dans la construction auctoriale. Ensuite, faire intervenir le lecteur dans la production littéraire actuelle. La place dérisoire que Golchiri cherche à accorder dans son texte au lecteur, peut être considérée, en effet, comme une tentative visant à faire de ce dernier une instance du texte à part entière. Néanmoins, ce procès s'achève par une tentative vaine pour attirer un lecteur complice envers cette communauté imaginée par l'auteur. Enfin, entre soupçon et invention, il s'agit d'un texte visant à écrire sur soi en sollicitant l'autre qui est dès le début revisité, par l'intermédiaire d'un destinataire ambigu qui est pour ainsi dire le double de l'auteur.

L'identité narrative plus ou moins fictionnelle, que compose Golchiri dans son œuvre, a donc valeur d'exorcisme. Elle est à la fois mise en scène et mise à distance d'un échec. Elle est figuration d'une impasse et du mouvement de sortie de cette impasse. À travers l'expérience toute personnelle de l'illégitimité initiale, à travers celles de l'insuffisance et de l'imposture, est examinée la question plus générale de la possibilité de passer du statut de lecteur à celui d'écrivain. À travers la relation problématique entre passé et présent, lecture et écriture, c'est la valeur de la littérature qui se trouve entre la double menace que font peser sur l'écriture valorisation et dévalorisation. Il n'est donc pas étonnant que ces interrogations aillent de pair, dans les textes qui les posent et les dépassent en même temps - car l'écriture, elle aussi, se prouve en écrivant - avec des choix qui situe Golchiri du côté de ses écritures ostensibles; écritures qui, à l'opposé des recommandations de Barthes du Degré zéro de l'écriture, assument le choix de donner à voir les signes de la "Littérature".

En effet, dans l'œuvre de Golchiri, la dualité entre les saisies proche et lointaine des personnages prend son essor. La saisie de loin de l'œuvre d'art ne saurait jamais coïncider avec sa saisie de près, au présent, de sorte que la stéréoscopie mise en place dans le texte golchirien constitue l'instrument qui 
donne à voir la non-coïncidence de ces deux saisies et le processus d'abolition de l'œuvre comme présent en œuvre, comme passé dans le cadre même de sa réception. Mais en même temps, le constat de cette dualité est de sortir de l'impasse constituée par le sentiment d'insuffisance découlant de la dé(ré)férence à un passé en ruine, et cela, en recréant un espace de jeu et de liberté entre une littérature du passé et une littérature comme passé, un espace référant toujours à une " écriture à (son) degré zéro » qui permette d'envisager une écriture au présent conciliable avec la conscience de la valeur des modèles et la prise en compte de ces modèles. Chacun des deux côtés de l'itinéraire fictionnel golchirien explore donc l'impasse où se manifeste la tentation d'une saisie anticipée de l'œuvre à venir comme passé. De ce fait, Golchiri met en scène le paradoxe de la valeur affirmée à l'œuvre par son abolition - l'œuvre comme l'acte créateur déjà en ruine — et la valeur qui lui est conférée selon laquelle l'œuvre est appréhendée comme lecteur/spectateur. 


\section{Bibliographie}

Allemand, Roger-Michel. Alain Robbe-Grillet. Paris: Seuil, 1997.

Allemand, Roger-Michel. Le Nouveau Roman. Paris: Ellipses, 1996.

Baetens, Jan et Dominique Viart. Ecritures contemporaines II. "États du roman contemporain: Actes du Colloque Calaceite 1996." Paris: Minard, 1999.

Barthes, Roland. Degré zéro de l'écriture - suivi de Nouveaux Essais critiques. Paris: Seuil, 1972.

Barthes, Roland. La chambre claire - note sur la photographie. Paris: Gallimard/Seuil (Cahiers du cinéma), 1980.

Barthes, Roland. Le plaisir du texte. Paris: Seuil, 1973.

Barthes, Roland. Roland Barthes par Roland Barthes. Paris: Seuil, 1975.

Barthes, Roland. S/Z. Paris: Seuil, 1970.

Cusset, François. Queer critics - La littérature française déshabillée par ses homolecteurs. Paris: P.U.F, 2002.

Deleuze, Gilles. Logique du sens. Paris: Minuit, 1969.

Deleuze, Gilles. Différence et répétition. Paris: P.U.F, 1968.

Derrida, Jacques. Marges - de la philosophie. Paris: Minuit, 1972.

Fages, Jean-Baptiste. Comprendre Jacques Lacan. Paris: Dunod, 2005.

Genette, Gérard. Figures III. Paris: Seuil, 1972.

Gide, André. Si le grain ne meurt. Paris: Gallimard, 1991.

Golchiri, Houchang. Jardin dans le jardin. Téhéran: Nilufar, 1999.

Golchiri, Houchang. Le Prince Ehtejab. Trad. Esmaili, Hossein et Jacques Selva avec l'avant-propos de Houchang Golchiri. Paris: L'Harmattan, 1990.

Lejeune, Philippe. Le pacte autobiographique. Paris: Seuil, 1996.

Merleau-Ponty, Maurice. La Prose du monde. Paris: Gallimard, 1969.

Nietzsche, Friedrich. Ainsi parlait Zarathoustra - un livre qui est pour tous et qui n'est pour personne. Trad. Maurice De Gandillac. Paris: Gallimard, 1971.

Ricœur, Paul. Soi-même comme un autre. Paris: Seuil, 1990

Robbe-Grillet, Alain. Les Derniers Jours de Corinthe. Paris: Minuit, 1994.

Robert, Marthe. Roman des origines et origines du roman. Paris: Gallimard, 1987.

Todorov, Tzvetan. "Les catégories du récit littéraire ». Communications 8.

«L'analyse structurale du récit ». Paris: Seuil. 131-57, 1981a.

Todorov, Tzvetan. Mikhaïl Bakbtine - le principe dialogique. Paris: Seuil, 1981 b. 